Скопје, Македонија

\title{
MODELS FOR THE LORENZ SYSTEM
}

\author{
BILJANA ZLATANOVSKA AND DONCO DIMOVSKI
}

\begin{abstract}
Using the systems of difference equations from [1] and [2], as approximation for the solutions of the Lorenz system of differential equations, we obtain two new systems of difference and differential equations as models for the Lorenz system. By computer simulations as in [3] and [4] we give examples, where locally the solutions of these new systems, approximate the solutions of the Lorenz system.
\end{abstract}

\section{INTRODUCTION}

Lorenz system is a nonlinear autonomous dynamic system whose explicit solutions are not known, but its behavior is studied extensively in the mathematical literature (for example [5], [6], [7], [8]).

The use of power series is one of the oldest methods for examining differential equations. It is used for numerical calculations and for theoretical results. In the literature there are numerous papers concerned with such a use of power series, like the papers [9], [10] and [11].

In [1] and [2] we have used power series combined with difference equations to find local approximations to the solutions of the Lorenz system of differential equations:

$$
\begin{aligned}
& \dot{x}=\sigma(y-x) \\
& \dot{y}=x(r-z)-y \\
& \dot{z}=x y-b z
\end{aligned}
$$

with parameters $\sigma, r, b$. For initial values $a_{0}=x(0), b_{0}=y(0), c_{0}=$ $z(0)$ by [12], [13], [14], we assuming the solutions of the system (1.1) are expanded as Maclaurin series,

$$
\begin{aligned}
& x(t)=a_{0}+a_{1} t+a_{2} \frac{t^{2}}{2 !}+\ldots+a_{n} \frac{t^{n}}{n !}+\ldots \\
& y(t)=b_{0}+b_{1} t+b_{2} \frac{t^{2}}{2 !}+\ldots+b_{n} \frac{t^{n}}{n !}+\ldots
\end{aligned}
$$

2010 Mathematics Subject Classification. 37N30, 41A58, 65L06.

Key words and phrases. Lorenz system, system of difference equations, system of differential equations. 


$$
z(t)=c_{0}+c_{1} t+c_{2} \frac{t^{2}}{2 !}+\ldots+c_{n} \frac{t^{n}}{n !}+\ldots
$$

By consecutive differentiation of (1.1), for the coefficients $a_{n}, b_{n}, c_{n}$ from (1.2), we obtain the following system of difference equations:

$$
\begin{aligned}
& a_{n}=\sigma\left(b_{n-1}-a_{n-1}\right) \\
& b_{n}=r a_{n-1}-b_{n-1}-\sum_{i=0}^{n-1}\left(\begin{array}{c}
n-1 \\
i
\end{array}\right) a_{i} c_{n-i-1} \\
& c_{n}=-b c_{n-1}+\sum_{i=0}^{n-1}\left(\begin{array}{c}
n-1 \\
i
\end{array}\right) a_{i} b_{n-i-1}
\end{aligned}
$$

In [1], starting from the representations (1.2) after mathematical transformations of (1.3) we obtained the following forms for the coefficients $a_{n}, b_{n}, c_{n}:$

$$
\begin{aligned}
& a_{n}=\tilde{a_{n}}+(-1)^{n-1} \sigma H_{n} \\
& b_{n}=\tilde{b_{n}}+(-1)^{n-1} W_{n} \\
& c_{n}=\tilde{c_{n}}+(-1)^{n-1}\left\{-b^{n} c_{0}\right\}
\end{aligned}
$$

where the parts $\tilde{a_{n}}, \tilde{b_{n}}, \tilde{c_{n}}$ are not given in explicit forms and the $H_{n}, W_{n}$ have the explicit forms:

$$
\begin{aligned}
H_{n}= & (\sigma+r)^{n-1}\left(b_{0}-a_{0}\right)+\sum_{m=1}^{\left[\frac{n-1}{2}\right]} \sum_{j=m+1}^{n-m}\left(\begin{array}{c}
n-j \\
m
\end{array}\right)\left(\begin{array}{c}
j-1 \\
m-1
\end{array}\right) \sigma^{n-j-1}\left(r^{j}-r^{m}\right) a_{0} \\
- & \sum_{m=1}^{\left[\frac{n}{2}\right]} \sum_{j=m}^{n-m}\left(\begin{array}{c}
n-j-1 \\
m-1
\end{array}\right)\left(\begin{array}{c}
j \\
m-1
\end{array}\right) \sigma^{n-j-1}\left(r^{j}-r^{m-1}\right) b_{0} \\
W_{n}= & {\left[\sigma+\left(r-c_{0}\right)\right]^{n-1}\left(a_{0}-b_{0}\right)\left(r-c_{0}\right)+\left[\left(r-c_{0}\right)^{n}-1\right] b_{0} } \\
- & \sum_{m=1}^{\left[\frac{n}{2}\right]} \sum_{j=m+1}^{n-m+1}\left(\begin{array}{c}
n-j \\
m-1
\end{array}\right)\left(\begin{array}{c}
j-1 \\
m-1
\end{array}\right) \sigma^{n-j}\left[\left(r-c_{0}\right)^{j}-\left(r-c_{0}\right)^{m}\right] a_{0} \\
& +\sum_{m=2}^{\left[\frac{n+1}{2}\right]} \sum_{j=m}^{n-m+1}\left(\begin{array}{c}
n-1-j \\
m-2
\end{array}\right)\left(\begin{array}{c}
j \\
m-1
\end{array}\right) \sigma^{n-j}\left[\left(r-c_{0}\right)^{j}-\left(r-c_{0}\right)^{m-1}\right] b_{0}
\end{aligned}
$$

Forgetting some parts from $\tilde{a_{n}}, \tilde{b_{n}}, \tilde{c_{n}}$ we obtained polynomials in explicit forms $\tilde{a_{n}}{ }^{\prime}, \tilde{b_{n}}{ }^{\prime}, \tilde{c_{n}}{ }^{\prime}$ Replacing $\tilde{a_{n}}, \tilde{b_{n}}, \tilde{c_{n}}$ in (1.4) by the polynomials $\tilde{a_{n}}{ }^{\prime},{\tilde{b_{n}}}^{\prime}, \tilde{c_{n}}{ }^{\prime}$, we obtained new coefficients $a_{n}(\approx), b_{n}(\approx), c_{n}(\approx)$ in explicit forms: 


$$
\begin{aligned}
& a_{n}(\approx)={\tilde{a_{n}}}^{\prime}+(-1)^{n-1} \sigma H_{n} \\
& b_{n}(\approx)={\tilde{b_{n}}}^{\prime}+(-1)^{n-1} W_{n} \\
& c_{n}(\approx)={\tilde{c_{n}}}^{\prime}+(-1)^{n-1}\left\{-b^{n} c_{0}\right\}
\end{aligned}
$$

In [2], using the coefficients $a_{n}(\approx), b_{n}(\approx), c_{n}(\approx)$ with initial values $a_{0}=$ $x(0), b_{0}=y(0), c_{0}=z(0)$ we obtained the system of difference equations

$$
\begin{aligned}
\mathbf{a}_{\mathbf{n}}(\approx) & =-A a_{n-1}(\approx)+B a_{n-2}(\approx)-C a_{n-3}(\approx)+D a_{n-4}(\approx)+ \\
& +(-1)^{n-5} \sigma\left\{H_{n}-A H_{n-1}-B H_{n-2}-C H_{n-3}-D H_{n-4}\right\}, \quad n>7 \\
\mathbf{b}_{\mathbf{n}}(\approx) & =-A b_{n-1}(\approx)+B b_{n-2}(\approx)-\bar{C} b_{n-3}(\approx)+D b_{n-4}(\approx)+ \\
& +(-1)^{n-5}\left\{W_{n}-A W_{n-1}-B W_{n-2}-\bar{C} W_{n-3}-D W_{n-4}\right\}, \quad n>6 \\
\mathbf{c}_{\mathbf{n}}(\approx) & =-A c_{n-1}(\approx)+B c_{n-2}(\approx)-C c_{n-3}(\approx)+D c_{n-4}(\approx)+ \\
& +(-1)^{n-5}\left[-b^{n-4} c_{0}\right]\left\{b^{4}-A b^{3}-B b^{2}-C b-D\right\}, \quad n>5
\end{aligned}
$$

where $A=1+\sigma+b, B=\sigma\left(r-c_{0}\right)-a_{0}^{2}, C=\sigma a_{0} b_{0}, \bar{C}=\sigma a_{0} b_{0}-\sigma b c_{0}, D=$ $-\sigma^{2} b_{0}^{2}$.

The coefficients $a_{p}(\approx), b_{q}(\approx), c_{s}(\approx)$ for $p \in\{1,2,3,4,5,6,7\}, q \in\{1,2,3,4,5,6\}, s \in$ $\{1,2,3,4,5\}$ are calculated directly from the system $(1.3)$ as the exact values $a_{p}(\approx)=a_{p}=x^{(p)}(0), b_{q}(\approx)=b_{q}=y^{(q)}(0), c_{s}(\approx)=c_{s}=z^{(s)}(0)$ and they are:

$$
\begin{aligned}
& \mathbf{a}_{\mathbf{1}}=\sigma\left(b_{0}-a_{0}\right) ; \mathbf{b}_{\mathbf{1}}=r a_{0}-b_{0}-a_{0} c_{0} ; \quad \mathbf{c}_{\mathbf{1}}=-b c_{0}+a_{0} b_{0} \\
& \mathbf{a}_{\mathbf{2}}=\sigma\left(b_{1}-a_{1}\right) ; \mathbf{b}_{\mathbf{2}}=r a_{1}-b_{1}-\sum_{i=0}^{1}\left(\begin{array}{l}
1 \\
i
\end{array}\right) a_{i} c_{1-i} ; \mathbf{c}_{\mathbf{2}}=-b c_{1}+\sum_{i=0}^{1}\left(\begin{array}{l}
1 \\
i
\end{array}\right) a_{i} b_{1-i} \\
& \mathbf{a}_{\mathbf{3}}=\sigma\left(b_{2}-a_{2}\right) ; \mathbf{b}_{\mathbf{3}}=r a_{2}-b_{2}-\sum_{i=0}^{2}\left(\begin{array}{l}
2 \\
i
\end{array}\right) a_{i} c_{2-i} ; \mathbf{c}_{\mathbf{3}}=-b c_{2}+\sum_{i=0}^{2}\left(\begin{array}{l}
2 \\
i
\end{array}\right) a_{i} b_{2-i} \\
& \mathbf{a}_{\mathbf{4}}=\sigma\left(b_{3}-a_{3}\right) ; \mathbf{b}_{\mathbf{4}}=r a_{3}-b_{3}-\sum_{i=0}^{3}\left(\begin{array}{l}
3 \\
i
\end{array}\right) a_{i} c_{3-i} ; \mathbf{c}_{\mathbf{4}}=-b c_{3}+\sum_{i=0}^{3}\left(\begin{array}{l}
3 \\
i
\end{array}\right) a_{i} b_{3-i} \\
& \mathbf{a}_{\mathbf{5}}=\sigma\left(b_{4}-a_{4}\right) ; \mathbf{b}_{\mathbf{5}}=r a_{4}-b_{4}-\sum_{i=0}^{4}\left(\begin{array}{l}
4 \\
i
\end{array}\right) a_{i} c_{4-i} ; \mathbf{c}_{\mathbf{5}}=-b c_{4}+\sum_{i=0}^{4}\left(\begin{array}{l}
4 \\
i
\end{array}\right) a_{i} b_{4-i} \\
& \mathbf{a}_{\mathbf{6}}=\sigma\left(b_{5}-a_{5}\right) ; \mathbf{b}_{\mathbf{6}}=r a_{5}-b_{5}-\sum_{i=0}^{5}\left(\begin{array}{l}
5 \\
i
\end{array}\right) a_{i} c_{5-i} \\
& \mathbf{a}_{\mathbf{7}}=\sigma\left(b_{6}-a_{6}\right)
\end{aligned}
$$




\section{Models For the Lorenz System}

For $c_{0}=z(0)=0$ in (1.5) and (1.7) we obtain: $C=\sigma a_{0} b_{0}=\bar{C}, \bar{B}=$ $\sigma r-a_{0}^{2}$ and

$$
\begin{aligned}
\bar{W}_{n} & =(\sigma+r)^{n-1}\left(a_{0}-b_{0}\right) r+\left(r^{n}-1\right) b_{0} \\
& -\sum_{m=1}^{\left[\frac{n}{2}\right]} \sum_{j=m+1}^{n-m+1}\left(\begin{array}{c}
n-j \\
m-1
\end{array}\right)\left(\begin{array}{c}
j-1 \\
m-1
\end{array}\right) \sigma^{n-j}\left(r^{j}-r^{m}\right) a_{0} \\
& +\sum_{m=2}^{\left[\frac{n+1}{2}\right]} \sum_{j=m}^{n-m+1}\left(\begin{array}{c}
n-1-j \\
m-2
\end{array}\right)\left(\begin{array}{c}
j \\
m-1
\end{array}\right) \sigma^{n-j}\left(r^{j}-r^{m-1}\right) b_{0}
\end{aligned}
$$

If we take $H_{n}^{*}=(-1)^{n-5} \sigma H_{n}, W_{n}^{*}=(-1)^{n-5} \bar{W}_{n}$ in the system (1.7) we obtain the following system of difference equations

$$
\begin{array}{rlrl}
a_{n}(\approx) & =-A a_{n-1}(\approx)+\bar{B} a_{n-2}(\approx)-C a_{n-3}(\approx)+D a_{n-4}(\approx) & & \\
& +H_{n}^{*}-A H_{n-1}^{*}-\bar{B} H_{n-2}^{*}-C H_{n-3}^{*}-D H_{n-4}^{*}, & & n>7 \\
b_{n}(\approx) & =-A b_{n-1}(\approx)+\bar{B} b_{n-2}(\approx)-C b_{n-3}(\approx)+D b_{n-4}(\approx) & \\
& +W_{n}^{*}-A W_{n-1}^{*}-\bar{B} W_{n-2}^{*}-C W_{n-3}^{*}-D W_{n-4}^{*}, & & n>6 \\
c_{n}(\approx) & =-A c_{n-1}(\approx)+\bar{B} c_{n-2}(\approx)-C c_{n-3}(\approx)+D c_{n-4}(\approx), & & n>5
\end{array}
$$

with the initial values $a_{0}=x(0), b_{0}=y(0), c_{0}=z(0)=0$ and (1.8).

Moreover, $H_{n}^{*}, W_{n}^{*}$ satisfy the system of difference equations

$$
\begin{aligned}
H_{n}^{*} & =\sigma\left(W_{n-1}^{*}-H_{n-1}^{*}\right) \\
W_{n}^{*} & =r H_{n-1}^{*}-W_{n-1}^{*}
\end{aligned}
$$

The systems (2.1) and (2.2) with the parameters $\sigma, r, b$ and the initial values $a_{0}=x(0), b_{0}=y(0), c_{0}=z(0)=0$ and $(1.8)$, produce the following system of difference equations for $a_{n}(\approx), b_{n}(\approx), c_{n}(\approx)$ :

$$
\begin{array}{rlr}
\mathbf{a}_{\mathbf{n}}(\approx) & =\sigma\left(b_{n-1}(\approx)-a_{n-1}(\approx)\right)+A \sigma\left(b_{n-2}(\approx)-a_{n-2}(\approx)\right) & \\
& -\bar{B} \sigma\left(b_{n-3}(\approx)-a_{n-3}(\approx)\right)+C \sigma\left(b_{n-4}(\approx)-a_{n-4}(\approx)\right) & \\
& -D \sigma\left(b_{n-5}(\approx)-a_{n-5}(\approx)\right)-A a_{n-1}(\approx)+\bar{B} a_{n-2}(\approx) & \\
& -C a_{n-3}(\approx)+D a_{n-4}(\approx), & n>7 \\
\mathbf{b}_{\mathbf{n}}(\approx) & =\left(r a_{n-1}(\approx)-b_{n-1}(\approx)\right)+A\left(r a_{n-2}(\approx)-b_{n-2}(\approx)\right) & \\
& -\bar{B}\left(r a_{n-3}(\approx)-b_{n-3}(\approx)\right)+C\left(r a_{n-4}(\approx)-b_{n-4}(\approx)\right) & \\
& -D\left(r a_{n-5}(\approx)-b_{n-5}(\approx)\right)-A b_{n-1}(\approx)+\bar{B} b_{n-2}(\approx) & \\
& -C b_{n-3}(\approx)+D b_{n-4}(\approx), & n>6 \\
\mathbf{c}_{\mathbf{n}}(\approx) & =-A c_{n-1}(\approx)+\bar{B} c_{n-2}(\approx)-C c_{n-3}(\approx)+D c_{n-4}(\approx), & n>5
\end{array}
$$


We say that the system (2.3) is a difference equations model for the Lorenz system.

Notation: In [3], this system of difference equations (2.3) is used as an initial system for constructing new systems of differential equations. In this paper, we give the procedure for obtaining it as well as the computer simulations for its local behavior.

If we look at the system (2.3) as a system of differential equations we obtain the following system:

$$
\begin{aligned}
\mathbf{x}^{(\mathbf{8})} & =\sigma\left(y^{(7)}-x^{(7)}\right)+A \sigma\left(y^{(6)}-x^{(6)}\right)-\bar{B} \sigma\left(y^{(5)}-x^{(5)}\right)+C \sigma\left(y^{(4)}-x^{(4)}\right) \\
& -D \sigma\left(y^{(3)}-x^{(3)}\right)-A x^{(7)}+\bar{B} x^{(6)}-C x^{(5)}+D x^{(4)} \\
\mathbf{y}^{(\mathbf{7})} & =\left(r x^{(6)}-y^{(6)}\right)+A\left(r x^{(5)}-y^{(5)}\right)-\bar{B}\left(r x^{(4)}-y^{(4)}\right)+C\left(r x^{(3)}-y^{(3)}\right) \\
& -D\left(r x^{(2)}-y^{(2)}\right)-A y^{(6)}+\bar{B} y^{(5)}-C y^{(4)}+D y^{(3)} \\
\mathbf{z}^{(6)} & =-A z^{(5)}+\bar{B} z^{(4)}-C z^{(3)}+D z^{(2)}
\end{aligned}
$$

with parameters $\sigma, r, b$ and initial values $a_{0}=x(0), b_{0}=y(0), c_{0}=z(0)=0$ and (1.8).

We say that the system (2.4) is a differential equations model for the Lorenz system.

The definition of the system (2.4) directly implies that the coefficients in the Maclaurin series for the solutions of the system (2.4) satisfy the system (2.3).

The system (2.4) is a linear system and its behavior is far away from the behavior of the Lorenz system. But, we have the opinion, that the local behavior of the system (2.4) is a good model for the local behavior of the Lorenz system.

\section{Computer Simulations of the Local Behavior}

In this section, we will give local behavior for the system of difference equations (2.3) and for the system of differential equations (2.4). At this moment the question of what conditions would imply the convergence of these power series is open.

The system (2.3): For given parameters $\sigma, r, b$ and initial values $a_{0}, b_{0}, c_{0}=$ 0 and (1.8), and a positive integer $m$, we consider the polynomials:

$$
\begin{aligned}
& P_{m}\left(a_{0}, b_{0}, c_{0}\right)(t)=a_{0}(\approx)+a_{1}(\approx) t+a_{2}(\approx) \frac{t^{2}}{2 !}+\cdots+a_{m}(\approx) \frac{t^{m}}{m !} \\
& Q_{m}\left(a_{0}, b_{0}, c_{0}\right)(t)=b_{0}(\approx)+b_{1}(\approx) t+b_{2}(\approx) \frac{t^{2}}{2 !}+\cdots+b_{m}(\approx) \frac{t^{m}}{m !} \\
& R_{m}\left(a_{0}, b_{0}, c_{0}\right)(t)=c_{0}(\approx)+c_{1}(\approx) t+c_{2}(\approx) \frac{t^{2}}{2 !}+\cdots+c_{m}(\approx) \frac{t^{m}}{m !}
\end{aligned}
$$

Let $T$ be a positive real number. We define the functions $x_{T}(t), y_{T}(t), z_{T}(T)$ for $t \in[0, \infty)$ as follows: $x_{T}(t)=P_{m}\left(a_{0}, b_{0}, c_{0}\right)(t), y_{T}(t)=Q_{m}\left(a_{0}, b_{0}, c_{0}\right)(t), z_{T}(t)=$ 
$R_{m}\left(a_{0}, b_{0}, c_{0}\right)(t)$, for $t \in[0, T]$. Next, we continue by induction. We assume that $x_{T}(t), y_{T}(t), z_{T}(t)$ are defined for $t$ in $[0, k T]$. We extend them on $[0,(k+1) T]$ by defining them for $t \in[k T,(k+1) T]$ :

$$
\begin{aligned}
& x_{T}(t)=P_{m}\left(a_{0}, b_{0}, c_{0}\right)(t-k T) \\
& y_{T}(t)=Q_{m}\left(a_{0}, b_{0}, c_{0}\right)(t-k T) \\
& z_{T}(t)=R_{m}\left(a_{0}, b_{0}, c_{0}\right)(t-k T)
\end{aligned}
$$

where $a_{0}=x_{T}(k T), b_{0}=y_{T}(k T), c_{0}=z_{T}(k T)$ and the initial values $a_{p}, b_{q}, c_{s}$ for $p \in\{1,2,3,4,5,6,7\}, q \in\{1,2,3,4,5,6\}, s \in\{1,2,3,4,5\}$ are calculated by (1.8).

The system (2.4): For given parameters $\sigma, r, b$, the procedure for looking at the local behavior of the system (2.4) is as follows. Let $T$ be a positive real number.

We take the solutions $x(t), y(t), z(t)$ for $t \in[0, T]$ of the system (2.4) obtained by the program Mathematica, for the initial values $a_{0}=x(0), b_{0}=$ $y(0), c_{0}=z(0)=0$ and (8). Let $\bar{x}_{T}(t)=x(t), \bar{y}_{T}(t)=y(t), \bar{z}_{T}(t)=z(t)$ for $t \in[0, T]$. Next, we continue in the same manner as for the system (2.3).

Let $\bar{x}_{T}(t), \bar{y}_{T}(t), \bar{z}_{T}(t)$ be defined for $t \in[0, k T]$. We extend them on $[0,(k+1) T]$ by defining them for $t \in[k T,(k+1) T]$ as follows. We take the solutions $x(t), y(t), z(t)$ for $t \in[0, T]$ of the system (2.4) obtained by the program Mathematica, for the initial values $a_{0}=x(0)=$ $\bar{x}_{T}(k T), b_{0}=y(0)=\bar{y}_{T}(k T), c_{0}=z(0)=\bar{z}_{T}(k T)$ and $a_{p}, b_{q}, c_{s}$ for $p \in$ $\{1,2,3,4,5,6,7\}, q \in\{1,2,3,4,5,6\}, s \in\{1,2,3,4,5\}$ calculated by (1.8), for these $a_{0}=x(0), b_{0}=y(0), c_{0}=z(0)=0$ Then, we define:

$$
\bar{x}_{T}(t)=x(t-k T), \bar{y}_{T}(t)=y(t-k T), \bar{z}_{T}(t)=z(t-k T)
$$

In examples, by computer calculations, for given parameters and initial conditions, for small values of $T$, we obtain the functions $x_{T}(t), y_{T}(t), z_{T}(t)$ (for the system (2.3)) and the functions $\bar{x}_{T}(t), \bar{y}_{T}(t), \bar{z}_{T}(t)$ (for the system (2.4)). We compare these solutions with the solutions of the Lorenz system (1.1), for the same parameters and initial conditions, obtained by the program Mathematica, and we obtain that they are close. So, it seems that the systems (2.3) and (2.4) are good models for the local behavior of the Lorenz system.

Example 1: Parameters $\sigma=5, r=25, b=0,8$; the initial values $a_{0}=0, b_{0}=1, c_{0}=0$ and time interval $[0,8]$.

Figure 2 and figure 3 show that the local behavior of the systems (2.3) and (2.4) for a small time step is close to the behavior the Lorenz system (fig.1). 


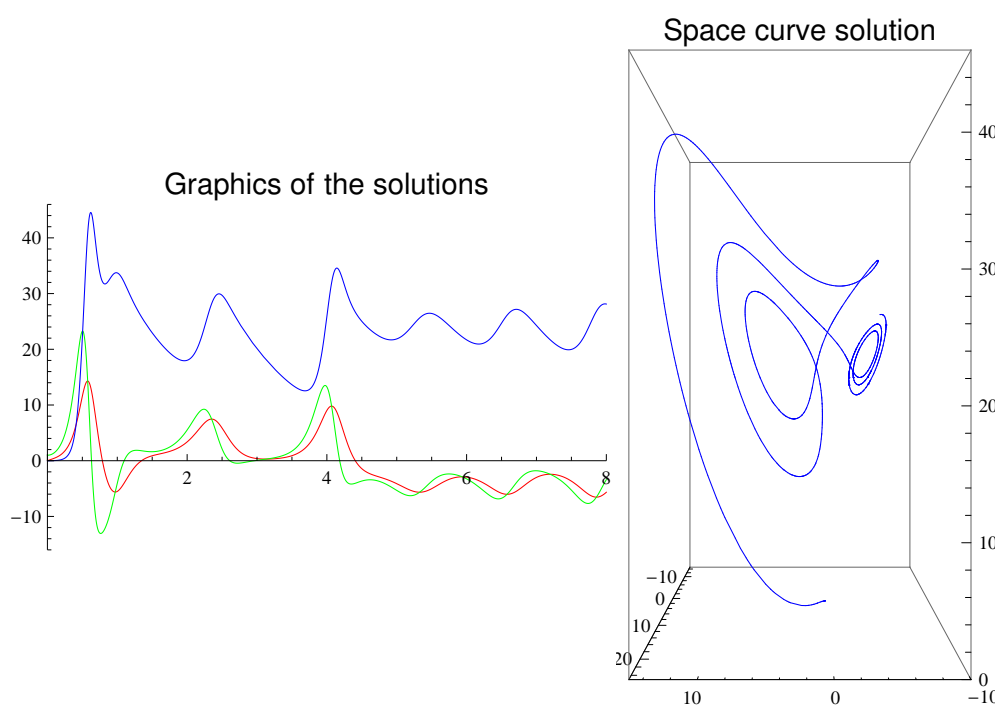

FiguRE 1. Results obtained by the program Mathematica for the system (1.1)

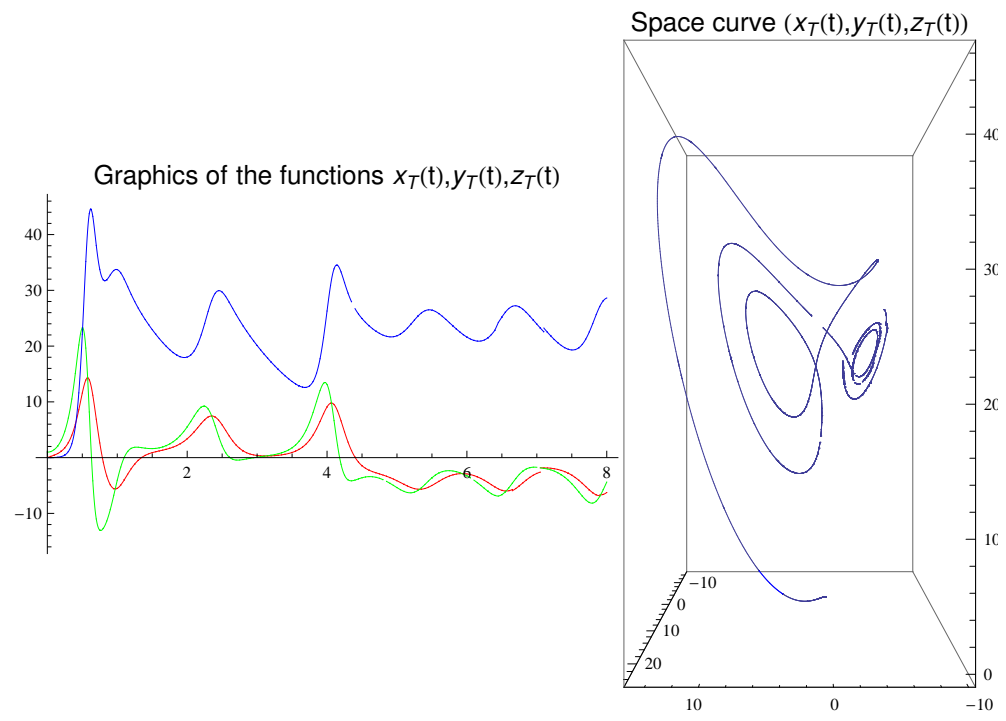

Figure 2. The solutions $x_{T}(t), y_{T}(t), z_{T}(t)$ for the sistem (2.3), for $m=15$ and $T=0,05$.

Example 2: Parameters $\sigma=10, r=23, b=5$; the initial values $a_{0}=$ $-2, b_{0}=3, c_{0}=0$ and time interval $[0,8]$. 


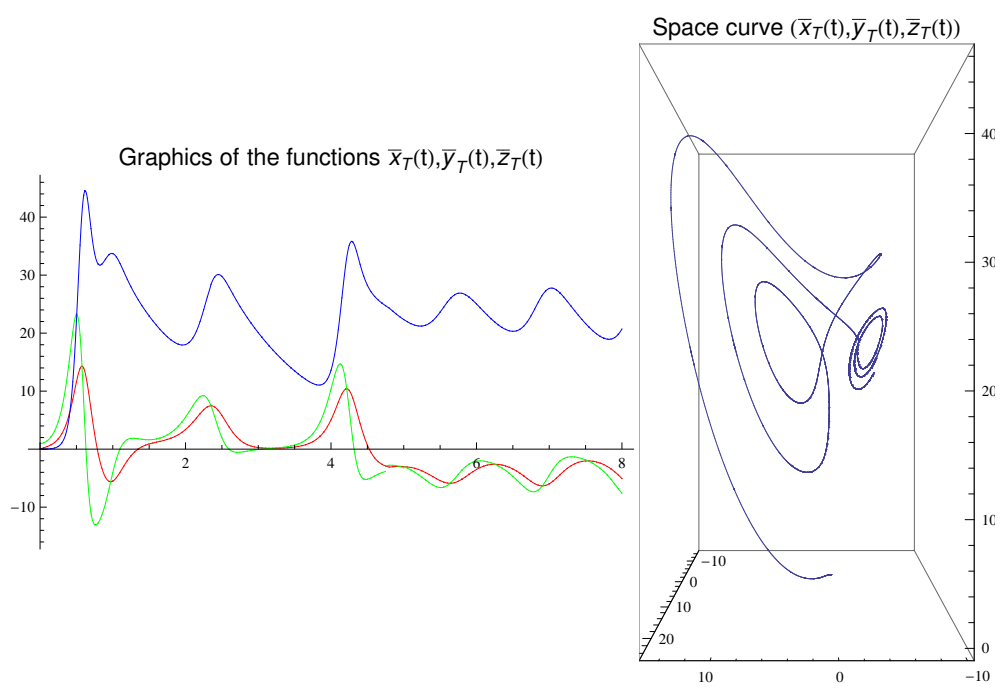

FiguRE 3 . The solutions $\bar{x}_{T}(t), \bar{y}_{T}(t), \bar{z}_{T}(t)$ for the sistem (2.4), for $T=0,05$.
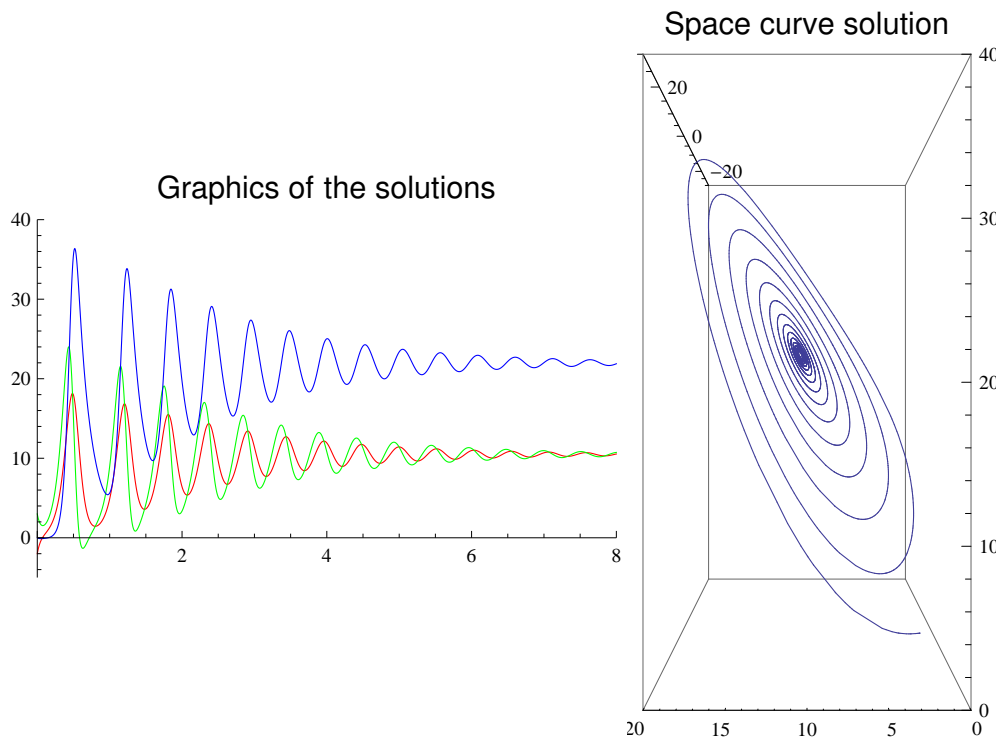

FigURE 4. Results obtained by the program Mathematica for the system (1.1)

Figure 5 and figure 6 show that the local behavior of the systems (2.3) and (2.4) for a small time step is close to the behavior the Lorenz system (fig.4). 


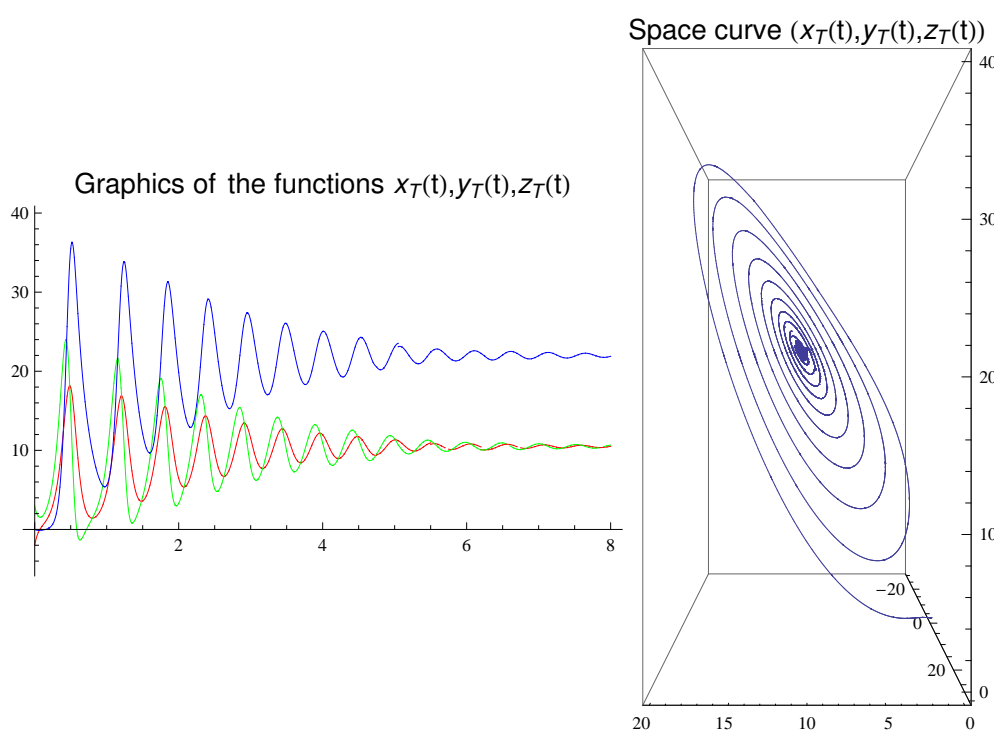

Figure 5. The solutions $x_{T}(t), y_{T}(t), z_{T}(t)$ for the sistem (2.3), for $m=15$ and $T=0,05$.

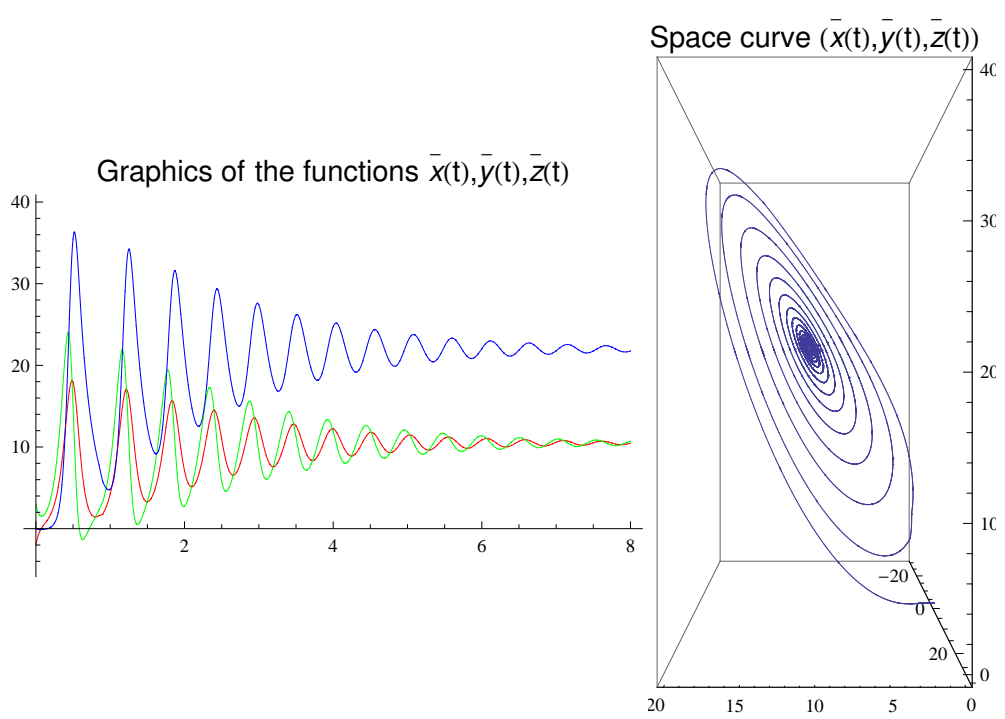

FiguRE 6 . The solutions $\bar{x}_{T}(t), \bar{y}_{T}(t), \bar{z}_{T}(t)$ for the sistem (2.4), for $T=0,05$.

\section{REFERENCES}

[1] B. Zlatanovska, D. Dimovski, Systems of difference equations approximating the Lorentz system of differential equations, - Contributions, Sec. Math. Tech. Sci., MANU, XXXIII, 1-2, ISSN 0351-3246 (2012) pp.75-96 
[2] B. Zlatanovska, D. Dimovski, Systems of difference equations as a model for the Lorentz system, Fifth International Scientific Conference FMNS - 2013,12-16.06.2013, Blagoevgrad, Bulgaria, Vol I, ISSN 1314 - 0272 (2013) pp.102-107

[3] B. Zlatanovska, Approximation for the solutions of Lorenz system with systems of differential equations, Bulletin Mathematique, No. 1, (ISSN 0351-336X - print, 18579914 - online) (2017) pp. 51-61

[4] B. Zlatanovska, D. Dimovski, Systems of differential equations approximating the Lorenz system, Proceedings of the 4th Conference of Mathematical Society of Moldova CMSM, Chisinau, Republic of Moldova, ISSN 978-9975-71-915-5, June 25-July 2 (2017) pp. 359-362

[5] K.T. Alligood, T.D. Sauer, J.A.Yorke, Chaos. An Introduction to Dynamical Systems, Springer-Verlag, New York, USA - Corrected third printing (2000) pp.359-370

[6] M.W. Hirsch, S. Smale, R.L.Devaney, An Differential Equations, Dynamical Systems and An Introduction to Chaos, Elsevier, USA (2004) pp.303-324

[7] S. Lynchi, Dynamical Systems with Applications using Mathematica, Boston, USA (2007)

[8] N. Razali, R. Ahmad, Solving Lorenz System by Using Runge-Kutta Method, European Journal of Scientific Research, Vol.32, No.2, ISSN 1450-216X (2009) pp.241-251

[9] R. Barrio, F. Blesa and M.Lara, VSVO Formulation of the Taylor Method for the Numerical Solution of ODEs, Comput. Math. Appl., 50 (2005) pp.93-111

[10] R. Barrio, Performance of the Taylor series method for ODEs/DAEs, Comput. Math. Appl.163, (2012) pp. 525-545

[11] M.A. Fathi, An Analytical Solution for the Modified Lorenz System, Proceedings of the World Congress on Engineering 2012, Vol I, (ISSN: 2078-0966 Online) London, U.K. (2012) pp.230-233

[12] M. Petrovic, Integration of differential equations by power series, Belgrade, (Serbian edition) (1938) pp.145-166

[13] J.E.Marsden and M. McCracken, The hopf bifurcation and its applications, SpringerVerlag, New York, USA (1976) pp.163-193

[14] L.S. Pontryagin, Ordinary differential equations, Science, Moscow, (Russian edition) (1970)

University "Goce Delcev",

FACUlty of Computer Sciences,

"Krste Misirkov" 10-A, P.O.Box 201, 2000 StiP

R. MACEDONIA

Email address: biljana.zlatanovska@ugd.edu.mk

University Ss. Cyril And Methodius,

Faculty of Natural Sciences and Mathematics,

"Karpos" 2 BB, P.O.Box 574, 1000 SKopje,

R. MACEDONIA

Email address: donco@pmf.ukim.mk 